\title{
Rétrospectives au bord de l'abîme : une lecture comparée de La mère, de Maxime Gorki, et 'Ta mère, de Bernardo Carvalho
}

\author{
Est-ce notre affaire, en somme, \\ Si tout retourne en poussière, \\ J'ai chanté sur tant d'abîme \\ Et vécu tant de miroirs. \\ Je ne suis pas un rêve, je n'apporte pas d'apaisement \\ Et encore bien le bonheur. \\ (Anna Akhmatova - Premier avertissement)
}

Résumé: Le présent travail vise à analyser la représentation de la figure maternelle dans l'œuvre la plus connue de l'auteur russe Maxime Gorki, La Mère, et le roman 'Ta mère de l'écrivain brésilien Bernardo Carvalho, publié en 2009. Dans cette étude, on prend en compte le contexte de production des deux œuvres afin de dégager les implications littéraires, politiques et culturelles ayant une incidence sur la représentation du réseau familial dans la société russe pendant le processus de lutte qui a abouti à la Révolution de 1917 (Gorki) et la récente guerre de Tchétchénie, l'axe du roman de Carvalho. Dans le même temps, en nous fondant sur ce roman, qui tourne autour du thème de la maternité, nous essayons d'envisager le sentiment paradoxal d'orphelinité qui marque le récit construit par l'auteur brésilien comme une métaphore permettant de comprendre non seulement la condition du monde post-utopique dans lequel nous vivons, mais aussi celle de l'écrivain qui tente en vain de construire une œuvre originale face à la tradition littéraire établie. ${ }^{1}$

Mots clés: Récit de filiation, littérature post-utopique, relations interculturelles, Bernardo Carvalho, Maxime Gorki.

Dans la littérature brésilienne, Bernardo Carvalho est peut-être l'écrivain qui cherche le plus à situer son œuvre sur un plan international. Pour cette raison, il ne faut pas s'étonner qu'il ait été l'un des auteurs invités à participer à la série de romans qui avait reçu le nom d'Amores expressos (Amours express), publiée par

1 Cet article a été réalisé grâce au soutien du CNPq au projet « Modernités alternatives : la littérature des BRICS (Brésil, Russie, Inde, Chine et Afrique du Sud) à l'ère de la mondialisation ».

2 Open Access. (c) 2021 Rachel Esteves Lima, published by De Gruyter. (c) BY-NC-ND This work is licensed under the Creative Commons Attribution-NonCommercial-NoDerivatives 4.0 International License. 
la maison d'éditions brésilienne Companhia das Lettras. Il s'agissait d'un projet qui a permis à dix-sept auteurs brésiliens de partir à l'étranger, visant à écrire une histoire d'amour après l'expérience d'un séjour d'un mois dans la ville de leur choix. Il revenait à Bernardo Carvalho d'écrire à partir d'une ville qui, depuis sa fondation, avait été la toile de fond de la production littéraire de grands écrivains : Saint-Pétersbourg. Confronté à cette tradition, qui réunit des noms comme Pouchkine, Gogol, Dostoïevski, parmi d'autres, l'auteur choisit de ne pas construire un récit excessivement métalinguistique, préférant pour l'essentiel traiter un thème relativement actuel, la guerre en Tchétchénie et les conflits inter-ethniques vécus sur le territoire russe post-soviétique. Comme l'écrivain lui-même l'a affirmé dans quelques interviews (2009a ; 2009b), il avait consacré son temps avant son départ à lire divers ouvrages historiques et journalistiques traitant de la situation politique en Russie, plus spécialement les livres d’Anna Politkovskaïa (2001; 2005 ; 2007), journaliste assassinée en 2006, très probablement en raison des critiques adressées au gouvernement Poutine et des stratégies de ce dernier en réaction aux mouvements séparatistes qui avaient fini par générer les deux guerres en Tchétchénie. C'est à partir de l'œuvre de Politkovskaiia que Carvalho va définir l'axe autour duquel son roman va évoluer : le rôle joué par les mères dans les conflits armés. Comme l'affirme, dans le roman, le personnage de Marina Bondareva, ellemême marquée par la mort d'un fils :

Les mères ont davantage à voir avec les guerres qu'elles n'imaginent. C'est le contraire de ce que tout le monde pense. Il ne peut pas y avoir de guerre sans mères. Plus que quiconque, les mères ont horreur de perdre. Nous sommes capables de tout pour éviter la mort d'un fils. Nous sommes capables de le défendre contre la justice elle-même. Les fils sont au-dessus de tout soupçon. Nous sommes capables de tuer pour un fils. Et nous finissons par être payées dans la même monnaie quand la guerre emporte un fils. Nous sommes prêtes à défendre notre progéniture et notre clan envers et contre tous. Sans vouloir comprendre que c'est de là que naissent les guerres. Tout le monde a une mère. Même la pire canaille, le pire bourreau. C’est indéniablement une sorte de fanatisme. (Carvalho 2010, 196)

Contrairement à ce que pourrait indiquer le titre portugais du roman de Carvalho - O filho da mãe (2009) [Le fils de la mère ou, dans son autre sens injurieux, Le fils de pute] -, il ne s'agit pas seulement de l'histoire d'un personnage, mais, principalement, d'un réseau de mères et de fils dont les vies sont meurtries par la guerre. Et comme cela arrive dans un autre roman de Carvalho, Neuf nuits (2005), les relations problématiques de parenté occupent encore le premier plan,

2 Le roman a été publié au Brésil en 2009. Dans les citations, nous avons utilisé la traduction française de Geneviève Leibrich, 'Ta mère, parue aux éditions Métailié en 2010. 
la figure du père persistant par son absence méritant d'être notée. Comme cela se passe pour les Indiens Kraho (de l'État brésilien du Tocantins) de Neufnuits, pour l'anthropologue nord-américain Buell Quain et aussi pour l'auteur de l'autofiction lui-même, qui a reçu un prestigieux prix littéraire brésilien, Rouslan et Andreï, les protagonistes de l'histoire qui a pour décor Saint-Pétersbourg, ne trouvent pas ici non plus dans la figure paternelle la protection nécessaire, ce qui les contraint à rechercher, en vain, l'appui de leurs mères, afin de survivre dans un monde dans lequel cohabiter avec les différences devient chaque fois plus difficile.

Rouslan est le fruit du mariage d'un Tchétchène avec une Pétersbourgeoise qui l'a abandonné au bout de deux mois à peine. Après la mort du père, il est élevé par sa grand-mère, à Grozny. Arrive alors la guerre en Tchétchénie, entraînant la persécution des jeunes de la région, tous considérés comme des rebelles séparatistes. Après avoir été séquestré par l'armée russe, corrompue, Rouslan est récupéré par sa grand-mère et finit par se réfugier en Ingouchie, où il apprend l'existence de sa mère, qu'il cherche à retrouver à Saint-Pétersbourg. Il obtient alors un emploi dans la restauration de façades, à la veille de la commémoration des 300 ans de la ville. Andreï, quant à lui, vient de la lointaine Vladivostok et se retrouve incorporé dans l'armée russe, institution que son beau-père l'avait obligé à intégrer, contre sa volonté et celle de sa mère, soumise à son mari. Là, il lui faut affronter les pratiques tortionnaires adoptées par ses supérieurs, et il se voit forcé à se prostituer par les officiers corrompus. La rencontre entre ces deux personnages, qui vivront l'histoire d'amour imposée par la série à laquelle appartient le roman, se fera quand Rouslan, à nouveau renié par sa mère, se transforme en pickpocket, l'une de ses victimes étant justement Andreï. Avec ce vol, ce dernier perd l'argent gagné lors de sa première passe avec un officier de l'armée, ce qui lui interdit le retour à la caserne. Pour s'en sortir, il va chercher de l'aide auprès du Comité des mères de soldats de Saint Pétersbourg, organisation humanitaire créée, selon Anna Politkovskaïa, pour « lutter pour la vie des soldats contre la machine à recruter de l'armée, qui les dévore ${ }^{3}$ » (Politkovskaya 2007, 86).

Le roman commence donc à partir de la rencontre de deux personnages au Comité de Saint-Péterbourg : Ioulia Stepanova et Marina Bondareva, anciennes amies d'école qui se retrouvent après que la première, même sans enfants, se sachant condamnée par un cancer, décide d'offrir son aide au Comité, où elle rencontre de façon inespérée Marina. Celle-ci, après avoir perdu son fils Pavel, qui s'est suicidé pour ne pas avoir à entrer dans l'armée russe, travaille comme volontaire au Comité et s'avère être la responsable qui secourt Andreï, pour qui le retour à la caserne est désormais impossible. À partir de la conversation entre

3 Toutes les traductions françaises des œuvres publiées en portugais sont de ma responsabilité. 
les deux amies, Bernardo Carvalho nous présente, en la mettant en relief, une des rares références littéraires figurant dans le roman : c'est pour répondre à la demande de la grand-mère de Ioulia qu'Anna Akhmatova aurait repris ses poèmes sur « les femmes et les mères qui attendaient leur mari et leur fils à l'extérieur des murs de la Kresty » (Carvalho 2010, 20), prison dans laquelle le fils d’Akhmatova fut détenu durant la période stalinienne. Tout comme la plus grande poétesse de Saint-Pétersbourg, dont le comportement éthique était devenu le modèle des écrivains s'opposant à l'oppression du régime communiste, Bernard Carvalho assume le récit de l'histoire des mères qui, même en temps de « démocratie », continuent de souffrir pour les fils dont la vie est en danger.

Bien que ce ne soit pas un roman autobiographique, sans doute peut-on faire le lien entre 'Ta mère, et même Neuf nuits, le roman que nous évoquions, et la production littéraire que le théoricien français Dominique Viart (2008) classe comme roman de filiation, une tendance fortement présente dans la littérature contemporaine. Dans ce type de récit, les relations familiales occupent le premier plan et au sein de ces relations, les personnages, qu'il s'agisse d'ailleurs d'une biographie ou non, cherchent mélancoliquement à lutter contre les lacunes identitaires qui les (dé)font. Comme l'affirme Alessandra Pajolla, « de telles œuvres exposent le parcours de personnages qui fouillent leurs origines à la recherche d'une espèce d'héritage reçu par testament, des sujets qui se sentent affectés par des circonstances liées à la généalogie et qui, de là, entreprennent des déplacements géographiques en quête d'autoconnaissance et d'appartenance » (Pajolla 2015). Cette sorte d'entreprise archéologique dans la littérature contemporaine va cependant indiquer bien davantage l'impossibilité de regrouper les fragments des identités fissurées que la perspective de leur recomposition. Dans ce parcours, la mémoire individuelle et collective se confondent et ce que l'on perçoit, c'est que les discours de filiation élargissent leur faisceau d'interrogations pour adresser, via l'histoire familiale, une critique aux projets téléologiques construits par la modernité, et parmi eux, ceux qui soutiennent, sur la base d'idéaux issus des Lumières, les concepts de formation et d'autonomie du littéraire. Autrement dit, les romans de filiation mettent en évidence la faillite non seulement des rôles historiquement construits par les membres de la famille patriarcale, mais aussi de l'utopie de la fraternité universelle à laquelle nous parviendrions par le développement des arts et des sciences, tant dans le monde capitaliste que dans le monde socialiste. Comme l'énonce Laurent Demanze :

Le récit de filiation semble ainsi de nos jours le symptôme d'une situation historique marquée par la lacune et l'inquiétude de la mémoire. Parents victimes d'une histoire devenue folle, pères évanouis ou mélancoliques, mères endeuillées ou sacrifiées: les figures parentales ne fournissent ni modèles ni repères à l'aune desquels se constituer. Le 
passé parental est le chapitre vacant de la mémoire, il est l'insu d'un sujet qui peine à le reconstituer à force d'hypothèses généalogiques et d'investigations imaginaires. Le passé se décline tour à tour en figures de l'héritage impossible, de la mémoire empêchée ou de la transmission d'une dette, comme si le rapport de l'individu contemporain à son passé était infailliblement frappé du sceau de la perte. Tout se passe comme s'il y avait eu une césure historique, et qui le laisse désorienté. (Demanze 2008)

En ce sens, nous aimerions faire, bien que brièvement, une lecture comparée du roman de Bernardo de Carvalho avec une œuvre qui n'y est pas explicitement citée, mais que l'on peut entrevoir dans le titre de son roman en portugais, $O$ filho da mãe [Le fils de la mère]. Nous défendons l'hypothèse qu'un tel titre, en raison de l'emploi du singulier, peut être interprété comme une référence au fameux roman de Maxime Gorki, écrit dans la décennie précédant la Révolution de 1917 et intitulé La mère. Comme on le sait, cette œuvre, qui ouvre le cycle de la littérature réaliste en Russie, est devenue une icône de la défense des idéaux révolutionnaires non seulement dans ce pays, mais partout dans le monde. On peut dire que l'histoire de la mère qui, instruite par son fils, se consacre sans restrictions à soutenir la lutte socialiste, agissant comme militante dans le groupe des révolutionnaires auquel elle appartient, se situe, dans l'œuvre de Carvalho, comme une ombre de plus, une vision fantasmatique de plus, parmi les nombreuses autres qui, depuis des siècles, interrogent le projet de modernisation et d'internationalisation qui présida à la construction de la ville de Saint-Pétersbourg ${ }^{4}$.

$\mathrm{Au}$ delà de la forte solidarité qui naît entre mère et fils, dans le roman de Gorki, l'idéal d'union transnationale pour l'émancipation des travailleurs est présenté sous des couleurs extrêmement idéalistes et romantiques par un des compagnons de lutte :

[...] Pour nous, il n'y a ni nations, ni races, il n'y a que des camarades ... et des ennemis. Tous les ouvriers sont nos amis, tous les riches, tous ceux qui détiennent l'autorité sont nos ennemis. Quand on regarde la terre avec de bons yeux, quand on voit combien nous, les ouvriers, nous sommes nombreux, quelle puissance spirituelle nous représentons, on a le cœur envahi de joie et de bonheur, comme si on célébrait une fête solennelle. Et le Français, et l'Allemand éprouvent le même sentiment, et les Italiens aussi se réjouissent. Nous sommes tous des enfants de la même mère, de la grande, de l'invincible fée de la fraternité des ouvriers, de tous les pays de la terre. Elle se développe, elle nous réchauffe de sa chaleur, c'est le second soleil au ciel de la justice ; et ce ciel est dans le cœur de l'ouvrier.

4 Sur les fantasmagories qui entourent le processus de la fondation de Saint-Pétersbourg, la ville prise comme emblématique pour la compréhension des paradoxes inhérents à la modernisation des pays sous-développés, à partir de l'étude de la littérature russe, voir les travaux de Solomon Volkov (1997) et Marshall Berman (2007). 
Quel qu'il soit, quelque nom qu'il se donne, le socialiste est notre frère en esprit, toujours, maintenant et à jamais, aux siècles des siècles. (Gorki 1945, 44)

Dans le roman $O$ filho da mãe, cependant, cette utopie est complètement démontée et les personnages de Bernardo Carvalho occupent les ruines de ce rêve de communion. S’opposent à présent à la Mère universelle, qu'il s'agisse de la mère biologique ou de la classe ouvrière internationale, des figures maternelles qui ne veulent ou ne peuvent venir en aide à leurs enfants : "Il y a mères et mères ", dira le personnage de Rouslan, faisant mouche (Carvalho, 2010, 169). De la même façon, les conflits entre communautés intra- et internationales se durcissent, aussi bien durant la période où Révolution de 1917 et Seconde guerre mondiale finirent par diviser le monde en instaurant la période appelée " Guerre froide », qu’après le processus de démocratisation de la Russie et la fin de l'Union soviétique. Les alliances entre étrangers et ethnies diverses donnent lieu, dans le roman, à une divergence d'expectatives, à l'abandon et à la mort, comme cela se passe dans l'histoire des protagonistes. Andreï, fils d'un Brésilien et d'une Russe de la lointaine Vladivostok, et Rouslan, fils d'un Tchétchène et d'une bourgeoise de Saint-Pétersbourg, sont victimes de pères et mères absents, et l'amour qui surgit entre eux est fondé tout autant sur le sentiment d'être orphelin et étranger dans la ville construite par Pierre le Grand que dans leur homosexualité, qui les situe encore plus en marge de la société. De toute évidence, le pessimisme qui accompagne normalement la prose de l'auteur du roman n'offre aucune échappatoire à ces êtres qui rompent avec la norme identitaire et qui n'ont pas de quoi assurer leur invisibilité dans une ville planifiée pour une surveillance totale. Comme cela est dit dans la lettre laissée par Andreï chez Marina, Saint-Pétersbourg est « la ville la plus artificielle de toutes. En trois siècles, on a essayé vainement de la nommer trois fois. Un nom par siècle. On a construit trois cents ponts, un pour chaque année, mais aucun ne mène nulle part. » (Carvalho 2010, 26)

Il est important de relever qu'à l'exemple de ce qui se passe dans les autres romans de Bernardo Carvalho, comme Mongolia (2004), Le Soleil se couche à São Paulo (2008), Reproduction (2015) et Sympathie pour le démon (2018), 'Ta mère met en relation une série de personnages de nationalités différentes, permettant aux lecteurs une interprétation de la façon dont s'opèrent les contacts interculturels dans le monde globalisé. De la sorte, nous pouvons accompagner la réalisation du désir de la mère de Rouslan d'abandonner sa ville et d'émigrer à New York, comme l'avait fait sa sœur, et assister à la transformation du père d'Andreï, ex-exilé politique de la dictature brésilienne aux confins de la Russie qui devient membre d'une organisation internationale de biopiratage, à l'extrême nord du Brésil. Dans le périmètre compris entre Belém, Oiapoque, la Guyane française et le Surinam, nous savons désormais quels sont processus globaux de corruption, de vol et d'ex- 
ploitation de main d'œuvre : un Indien achète des terres et engage des Céaranais sans emploi pour les faire travailler en régime de semi-esclavage dans les mines clandestines, pendant que Suisses et Français emploient des Brésiliens pour faire la contrebande de plantes médicinales amazoniennes destinées à la recherche scientifique de laboratoires européens. Et à la suite de ces contacts, un nombre croissant d'enfants orphelins sont dispersés à travers le monde.

$\mathrm{Au}$ contraire de ce que l'on pourrait supposer, la mondialisation aiguise les conflits sociaux et le récit de Carvalho les expose dans toute leur cruauté possible. Saint-Pétersbourg devient alors l'espace métaphorique pour interroger l'utopie du respect de la diversité, du multiculturalisme, si présent dans les discours de la contemporanéité. Ce sont l'irrespect des différences et la survivance du discours de la nation qui promeuvent la guerre en Tchétchénie ; c'est la lutte des classes qui empêche les parents de Rouslan de poursuivre leur relation ; c'est le préjugé qui pousse le beau-père d'Andreï à le faire entrer dans l'armée, où le garçon sensible qui parlait avec les murs devrait devenir un « homme »; c'est le racisme qui mène Maxime, le demi-frère skinhead de Rouslan, à le considérer comme un « cul-noir » (Carvalho 2010, 166), parce qu'il est Tchétchène, et à vouloir l'éliminer. Cela étant, on est chaque fois plus éloigné de la tradition ingouche qui, selon ce que nous apprend le roman, propose l'adoption par solidarité d'un kunak, " un étranger, ou un membre d'un autre clan ou d'une autre tribu, avec qui s'établit un pacte de protection et de fraternité » (Carvalho 2010, 44).

Selon notre interprétation, Bernardo Carvalho s'approprie la paranoïa qui hante les œuvres littéraires écrites sur Saint-Pétersbourg depuis Le Cavalier de bronze (2003 [1833]) ${ }^{5}$, le poème d'Alexandre Pouchkine, et ce, afin d'étendre l'univers de sa critique aux résultats du processus de modernisation opéré à un niveau global, une fois que la perte des référentiels éthiques, moraux, artistiques et identitaires s'avère omniprésente. La condition d'orphelinité devient alors le symptôme d'une ère dans laquelle toutes les révolutions sont frustrées, et le conflit entre peuples et générations prend chaque fois plus d'ampleur, nous laissant comme seule option de survivre dans un monde privé des modèles, $\mathrm{y}$ compris littéraires, à partir desquels nous aurions pu orienter nos actions et nos discours.

5 À la fin du célèbre poème, considéré par de nombreux critiques comme le plus grand de la littérature russe, Eugène, ayant perdu sa bien-aimée dans l'une des inondations qui ont dévasté Saint-Pétersbourg depuis sa fondation par Pierre le Grand, commence à se sentir persécuté par la statue du tsar, qu'il avait osé défier en un geste de désespoir. Le Cavalier de Bronze critique le processus de modernisation russe et influencera d'autres écrivains du pays dans leurs représentations des écarts entre les intérêts de l'État et ceux des pauvres. 
$\mathrm{Au}$ sens où l'entend Silviano Santiago (2014; 2015) lorsqu'il se réfère aux discours élaborés par les intellectuels brésiliens, nous vivons un moment dans lequel le paradigme de la «formation », pensé comme un projet qui nous ferait prendre exemple sur l'Occident pour construire notre autonomie en tant que nation, est en train de s'éteindre, le paradigme de l'insertion du Brésil dans l'ensemble des nations devant se substituer à lui. Nous considérons que les récits de Bernardo Carvalho font partie de cette entreprise, dès lors qu'ils déconstruisent toute possibilité de penser de façon isolée la situation politique, économique et culturelle du Brésil, ou même de tout autre pays, sans prendre en compte ses liens avec les évènements de l'espace global. Ainsi, ses romans, même lorsqu'ils se situent à l'étranger, nous offrent aussi, que nous soyons lecteurs brésiliens ou autres, une perspective de compréhension des problèmes qui nous concernent inévitablement, dans tous les domaines.

$\mathrm{Au}$ contraire des livres précédents de Carvalho, 'Ta mère a été écrit à la troisième personne, tout comme le roman réaliste de Gorki. Mais le roman se construit, au fond, comme un récit post-moderne et post-utopique, se présentant à nous comme une mise en garde contre l'intolérance qui domine le monde, génératrice des guerres et de la normalisation des identités. Les chimères auxquelles fait référence le roman sont encore éliminées par la crainte d'accueillir en soi les différences, comme cela arrive avec Rouslan et Andreï, et aussi comme ce petit veau ayant eu la malchance de venir au monde malformé et en dehors des normes génétiques établies. L'histoire d'amour du contrat éditorial se termine par conséquent sans futur, comme un autre « Requiem pour Saint-Pétersbourg », à ajouter à celui d'Anna Akhmatova', à la recherche d'un sens à donner à tout ce qui s'est passé dans cet espace-métaphore qui en dit tant sur la réalité que nous vivons tous. Il nous reste finalement à faire le deuil des modèles anciens auxquels nous nous identifions, y compris les romans qui ont ouvert le $\mathrm{XX}^{\mathrm{e}}$ siècle, confiants dans la capacité humaine de changer radicalement le cours de l'histoire. Et peut-être les récits contemporains peuvent-ils au moins nous aider dans ce travail. En fin de compte, comme l'affirme le personnage de Marina Bondareva :

Les histoires d'amour peuvent ne pas avoir d'avenir, mais elles ont toujours un passé. Voilà pourquoi les gens s'accrochent à tout ce qui les renvoie à ce qu'ils ont perdu. Les livres qu'ils lisent concernent toujours le passé. Les romans historiques, les mémoires, les biographies, tout doit être conçu dans un mode rétrospectif, sinon ça n’aurait aucun sens. Personne n’a

6 Euvre la plus connue de la poétesse, écrite entre 1935 et 1940, « Requiem » dépeint la terreur du régime stalinien et les jours passés à la porte de la prison de Leningrad, à attendre Lev Gumiliov, le fils qu'elle a eu avec le poète Nicolaï Gumiliov, condamné par le régime et mort en 1921. 
envie de lire ce qui va arriver, au bord de l'abîme. Les gens ont besoin de s'accrocher à ce qu'ils connaissent déjà. Les modernismes ne pouvaient pas durer. Ni les révolutions. (Carvalho 2010, 195-196)

\section{Bibliographie}

Akhmatova, Anna. Poèmes. Jeanne Rude (trad.). Paris : Seghers, 1968.

Akhmátova, Anna. Antologia Poética. Lauro Machado Coelho (éd. et trad.). Porto Alegre : L\&PM, 2009.

Berman, Marshall. Tudo que é sólido desmancha no ar: A aventura da modernidade. Carlos Felipe Moisés et Ana Moria L. Ioriatti (trad.). São Paulo : Companhia das Letras, 1982 [All That is Solid Melts Into Air. New York : Simon \& Schuster, 1982].

Carvalho, Bernardo. Mongolia. Geneviève Leibrich (trad.). Paris : Métailié, 2004 [Mongólia. São Paulo : Companhia das Letras, 2003].

Carvalho, Bernardo. Neuf nuits. Geneviève Leibrich (trad.). Paris : Métailié, 2005 [Nove noites. São Paulo : Companhia das Letras, 2002].

Carvalho, Bernardo. Le Soleil se couche à São Paulo. Geneviève Leibrich (trad.). Paris : Métailié, 2008 [O sol se põe em São Paulo. São Paulo : Companhia das Letras, 2007].

Carvalho, Bernardo. "A Rússia por trás de suas fachadas ». Antonio Gonçalves Filho (interview). <https://cultura.estadao.com.br/noticias/artes,a-russia-por-tras-de-suasfachadas.334828>. O Estado de S. Paulo, 6 mars 2009a (16 juin 2017).

Carvalho, Bernardo. "Pânico em São Petersburgo ». Bruno Dorigatti (interview). <https://blog. saraiva.com.br/panico-em-sao-petersburgo//>. 29 septembre 2009b (16 juin 2017).

Carvalho, Bernardo. 'Ta mère. Geneviève Leibrich (trad.). Paris : Métailié, 2010 [O filho da mãe. São Paulo : Companhia das Letras, 2009].

Carvalho, Bernardo. Reproduction. Geneviève Leibrich (trad.). Paris : Métailié, 2015 [Reprodução. São Paulo : Companhia das Letras, 2013].

Carvalho, Bernardo. Sympathie pour le démon. Danielle Schramm (trad.). Paris : Métailié, 2018 [Simpatia pelo demônio. São Paulo : Companhia das Letras, 2016].

Demanze, Laurent. «Récits de filiation ». <https://www.fabula.org/atelier.php?R\%26eacute \%3Bcits_de_filiation>. Fabula, 7 août 2008 (16 Juin 2017).

Gorki, Maxime. La Mère. Serge Persky (trad.). Paris : Éditions Hier et Aujourd’hui, 1945.

Pajolla, Alessandra Dalva de Souza. « Bastardos e órfãos contemporâneos: a arqueologia da infância nos romances de filiação. " Estudos de Literatura Brasileira Contemporânea 46 (2015). <www.scielo.br/scielo.php?script=sci_arttext\&pid=S2316-40182015000200105\& lng=pt\&tlng=pt $>$. Brasilia : UnB (16 Juin 2017).

Politkovskaya, Anna. A Dirty War: A Russian Reporter in Chechnya. John Crowfoot (trad.). Londres : Harvill, 2001.

Politkovskaya, Anna. Putin's Russia: Life in a Failing Democracy. Arch Tait (trad.). New York : Henry Holt \& Co, 2005.

Politkovskaya, Anna. Um Diário Russo. Nivaldo Montigelli Júnior (trad.). Rio de Janeiro : Rocco, 2007.

Pouchkine, Alexandre. Poltava - Le Cavalier de Bronze. Léonid et Nata Minor (trad.). Genève : Éditions des Syrtes, 2003 [1828/1833]. 
Santiago, Silviano. «Anatomia da formação ». Folha de S. Paulo. Caderno Ilustríssima, 7 septembre 2014.

Santiago, Silviano. «Silviano Santiago: A literatura brasileira precisa superar o paradigma da formação e entrar no da inserção ». Ewerton Martins Ribeiro (interview). <https://www. ufmg.br/online/arquivos/037483.shtml>. Belo Horizonte : UFMG, 2015 (10 Mars 2015).

Viart, Dominique. «Filiations littéraires ». États du roman contemporain. Jan Baetens et Dominique Viart (dir.). Écritures contemporaines 2 (1999) : 115-137.

Volkov, Solomon. São Petersburgo: uma História Cultural. Marcos Aarão Reis (trad.). Rio de Janeiro: Record, 1997 [St. Petersburg. A Cultural History. Antonina W. Bouis (trad.). New York : Free Press, 1995].

Rachel Esteves Lima is Professor of Brazilian Literature at the Federal University of Bahia (UFBA), with postdoctoral studies at the Paris XIII University (2011), a Ph.D. in Literary Studies/Comparative Literature (1997) at the Federal University of Minas Gerais (UFMG) and an M.A. in Literary Studies/Brazilian Literature (1987), also at UFMG. Co-editor of the books A Antropofagia na era da globalização (EDUFBA, 2016) and $O$ espaço biográfico: perspectivas interdisciplinares (EDUFBA, 2016). Coordinator of the Study Group on Criticism and Contemporary Culture (UFBA) and researcher in the project "Alternative Modernities: BRICS Literature in the Globalization Era", sponsored by the National Council for Scientific and Technological Development (CNPq). 